\title{
Determinación del estado de maduración de frutos de feijoa mediante un sistema de visión por computador utilizando información de color
}

\section{Ripeness determination in feijoa fruits by using a computer vision system and colour information}

Juan Pablo Bonilla-González'
Flavio Augusto Prieto-Ortiz

Recibido: enero 16 de 2016 Aceptado: abril 09 de 2016

\section{Resumen}

Determinar el estado de madurez de productos agrícolas, generalmente depende de un análisis realizado por expertos humanos. La decisión final sobre el estado de madurez donde se encuentra el producto, resulta de correlacionar algunas de sus propiedades físicas con características químicas e internas del fruto. La necesidad de preservar la integridad del fruto en dicho análisis, hace necesario la implementación de tecnologías que emitan un juicio sobre el estado del mismo, sin necesidad de destruirlo. El uso del índice de color como propiedad física, contribuye a la solución de este problema. En este documento, se presenta un sistema de visión por computador para clasificar en tres estados de madurez un fruto exótico específico, feijoa - Acca sellowiana-. Los resultados obtenidos a partir de la clasificación, utilizando diferentes clasificadores, permiten obtener una respuesta superior al 90\%, para 156 imágenes de frutos de feijoa utilizadas en el estudio.

Palabras clave: Acca sellowiana, feijoa, reconocimiento de patrones, sistema de visión por computador.

\begin{abstract}
Determine the ripeness of agricultural products generally depends on an analysis by human experts. The final decision on the state of maturity where the product is found, requires correlating some of their physical features with chemical and internal characteristics of the fruit. The need to preserve the integrity of the fruit on this analysis requires implementation of technologies to pass judgment on its condition without destroying it. The use of colour index, as physical property, contributes to solving this problem. In this document is presented a machine vision system to classify into three stages of maturity a specific exotic fruit: feijoa -Acca sellowiana-. The obtained classification using artificial intelligence tools, as these are artificial neural networks, have shown an adequate classification over 90\% from 156 images of Feijoa fruit used in the study.
\end{abstract}

Keywords: Acca sellowiana, feijoa, pattern recognition, computer vision system.

\footnotetext{
1 Ingeniero Mecatrónico, Universidad Nacional de Colombia. E-mail: jpbonillag@unal.edu.co

2 Ingeniero Electrónico, Doctor en Automática Industrial, Universidad Nacional de Colombia. E-mail: faprietoo@unal.edu.co
} 


\section{Introducción}

Los productos agrícolas, en especial las frutas, se diferencian por sus propiedades físico-químicas, como color, textura, sabor, olor, entre otras. Algunas características de tipo físico como tamaño y color, ayudan a determinar el estado de madurez de los frutos. De manera independiente, cada fruto sufre cambios fisiológicos con el paso del tiempo hasta llegar a la maduración, momento en el cual puede ser consumido en las mejores condiciones. Cada uno de los diferentes estados de madurez en los frutos posee procesos fisiológicos específicos; esto evidencia un indicador físico de fácil análisis por medio de inspección visual, el color.

Aunque es posible realizar esta clasificación por estado de madurez en laboratorios especializados mediante el análisis de parámetros físicos, como el color, y químicos, como la acidez, los procesos en los equipos usados para este fin suelen ser destructivos y no pueden ser aplicados directamente en el cultivo, sino en la etapa poscosecha del fruto. En consecuencia, el desarrollo de algoritmos de visión por computador orientados a la determinación en campo del estado de desarrollo de los frutos, se presenta como una posible solución de mejora en las prácticas en cosecha y poscosecha.

El objetivo de mejorar estos procesos se realiza con el fin de lograr una mayor y mejor comercialización de los productos, en los mercados tanto nacionales como internacionales (Bustamante-Zapata, Porto-Pérez, \& Hernández-Taboada, 2013). En especial, los frutos exóticos requieren que su oferta se haga en óptimas condiciones ya que no existen procesos normalizados para los mismos (Sanabria-Neira \& Puentes-Montañez, 2011). Por esto, en gran medida debido a su diversidad y condiciones especiales en general, hace que el mercado tenga que realizar la trazabilidad del producto para garantizar su calidad.

Las técnicas de visión artificial han sido ampliamente utilizadas en la identificación y análisis de imágenes (Cárdenas \& Prieto-Ortíz, 2015; Ce-
rón-Correa, Salazar-Jiménez, \& Prieto-Ortíz, 2013; Torres-Barahona, León-Medina, \& Torres-Díaz, 2012). En este sentido se propone un sistema de visión por computador para la identificación del estado de maduración de Feijoa -Acca sellowianade manera automática, a partir de una imagen. Mediante el uso de técnicas de adquisición y procesamiento de imágenes, segmentación, extracción de características sobre los puntos de interés $y$, finalmente, la aplicación de clasificadores del tipo bayesiano y red neuronal artificial.

Este artículo se divide en una fundamentación teórica que incluye el estado del arte en procesos de análisis de colorimetría y una descripción del sistema propuesto, utilizando técnicas de visión por computador, pasando por su justificación hasta su aplicación. Posteriormente se presentan los resultados obtenidos a partir del análisis de los diferentes espacios de color en los frutos de feijoa, comprobando su funcionamiento con diferentes clasificadores; finalmente se presentan las conclusiones del trabajo.

\section{Marco teórico y metodología}

\subsection{Feijoa y estado de maduración}

Una de las mejores características para describir el estado de madurez de los frutos, es el índice de color. Las variaciones en el color se producen por disminución de clorofila y aumento de pigmentos de color amarillo, naranja y rojo (García, García, Hernández, \& Pérez 2011). Este proceso de modificación de color en el caso de frutos como la feijoa, durante su proceso de madurez, se evidencia en modificaciones del tono verde, pasando de oscuro a claro.

La feijoa es un fruto característico de la región latinoamericana siendo cultivada en su mayoría en Brasil y Colombia. Por ser un fruto exótico es un producto de gran demanda en Europa y América del Norte, principalmente usado como agregado de bebidas $u$ otros productos alimenticios. Los frutos de feijoa poseen una capa de piel verde, 
que recubre otra capa de gránulos blancos, la cual contiene una pulpa con semillas y jalea, situada en una cavidad central formada por entre tres y cinco lóbulos (East, Trejo-Araya, Hertog, Nicholson, \& Mawson 2009). El periodo de cosecha es corto y por tanto, la necesidad de monitorear y conocer el estado real de madurez en el fruto es un aspecto esencial.

En las zonas rurales de Colombia la clasificación por estado de madurez de la feijoa se realiza evaluando algunos aspectos de su aspecto físico como la coloración en el fruto y la textura. Esta última, evaluándola ya sea de manera manual por medio del tacto, en campo, o midiendo su firmeza mediante pruebas de laboratorio. Los algoritmos de visión por computador y la determinación de parámetros físicos que contribuyan a una clasificación de los frutos, correlacionando los procesos químicos internos con lo evidenciado en aspectos como el índice de color, son en la actualidad un campo de investigación activo en el área agrícola (Thompson, 1998). Para frutos exóticos como la feijoa -Acca sellowiana- los estudios aún son menores, y las aplicaciones tecnológicas mínimas y poco implementadas.

Entre las aplicaciones de visión por computador para la evaluación de la calidad de los productos, las investigaciones se han centrado en la clasificación y predicción del estado de los frutos. Sin embargo la segmentación y selección más precisa de características genera nuevos tópicos de estudio como la identificación de los frutos en campo o la estimación de características fisicoquímicas (Abdullah, Mohamad-Saled, Fathinul-Syahir, \& Mohd-Azemi, 2006; Danti \& Suresha, 2012).

Así, la implementación, uso y caracterización de modelos, hace que cada nuevo desarrollo sea único y particular. Esto, debido a que las frutas, verduras y granos suelen presentarse al consumidor final en lotes donde la homogeneidad y la apariencia de estos tienen un efecto radicalmente significativo en el consumidor. Por esta razón la presentación de los productos agrícolas está orientada a la limpieza y clasificación del producto durante todas sus etapas, hasta llegar al consumidor final.

La identificación del color en los frutos por medio de visión por computador ha sido también una rama de gran interés (Blasco, Aleixos, \& Moltó 2003; Du \& Sun, 2006; Kondo, Ahmad, Monta, \& Murase, 2000; Ohali, 2011; Sandoval, 2005; Torres, Montes, Pérez, \& Andrade 2013; Vignoni, Cesari, Forte, \& Mirabile, 2006). Esto ha sido posible a partir de la separación de planos, por ejemplo del espacio de color RGB (por sus siglas en inglés Red, Green y Blue), con el fin de identificar cada una de las variaciones a lo largo del proceso de maduración.

Desde 1992, cuando se presentó uno de los primeros sistemas de análisis de imagen con el fin de evaluar el color de frutos de melocotón, ha surgido la necesidad de evaluar el color en otras variables, cuantificando la luminosidad y/o las variaciones entre los tonos de color, surgiendo así otros espacios de color con los cuales fuese posible realizar este análisis. El color es una de las características fundamentales para la percepción visual de los frutos, la cual es posible por medio de imágenes digitales. De esta manera, es posible diseñar y desarrollar sistemas automáticos que identifiquen los colores externos del fruto y estimen en función de este, el estado de madurez (Arivazhagan, Newlin, Selva, \& Ganesan, 2010). Sin embargo, el desarrollo de estos sistemas es complejo en frutas donde el color es uniforme durante todo su proceso de maduración, como en el caso de la feijoa.

\subsection{Espacios de color}

En este trabajo son usados cuatro modelos o espacios de color para definir el color: el espacio RGB, CIELab, YCbCr (por la abreviatura de Luma, Crominancia de azul y Crominancia de rojo), y HSV (por sus siglas en inglés Hue, Saturation y Value). En los métodos y técnicas de visión por computador, varios tipos de cámaras en el espectro visible, infrarrojo u otras longitudes de onda en el espectro electromagnético, son usadas ampliamente para 
adquirir imágenes, en función de la aplicación. Las cámaras de color RGB son utilizadas comúnmente en muchas industrias, debido principalmente a la facilidad en el análisis de imagen y su bajo costo (Manickavasagana, Al-Mezeinia, \& Al-Shekaili, 2014).

El espacio de color $L^{*} a{ }^{*} b^{*}$ o CIELab, es una norma internacional para la medición de color, adoptado por la Comisión Internationale d'Eclairage, CIE, en 1976. $L^{*}$ es la componente de luminancia en el rango de 0 a 100, y los parámetros a* (de verde a rojo) y b* (de azul a amarillo) son los dos componentes cromáticos, que varían entre -120 y 120 (Yama \& Papadakis, 2004). Aunque existen diferentes espacios de color, uno de los más utilizados en la medición del color en alimentos (frutas, hortalizas y granos) es el espacio de color $L^{*} a^{*} b^{*}$ debido a la distribución uniforme de los colores y, también a que está muy cerca de la percepción humana del color. Existen en la actualidad soluciones comerciales (colorímetros) para obtener las medidas del espacio de color $L^{*} a^{*} b^{*}$, pero estos miden generalmente en pequeñas áreas, lo que resulta en áreas no representativas de unos pocos centímetros cuadrados (León, Mery, Pedreschi, \& León, 2006).

Por su parte, el modelo de color $\mathrm{YCbCr}$ posee un formato en el cual la información de luminancia es representada por un componente único $(\mathrm{Y})$ y la información de color es descompuesta en dos diferentes componentes de color, $(\mathrm{Cb}$ - Crominancia de diferencia de azul y $\mathrm{Cr}$ - Crominancia de diferencia de rojo). Por último, el espacio de color HSV proporciona información de la imagen en tres distintos componentes $\mathrm{H}$ (Matiz o Hue por sus siglas en inglés), S (Saturación) y V (Brillo o Value por sus siglas en inglés).

\subsection{Clasificadores}

Los clasificadores son esenciales en el desarrollo de sistemas de visión por computador, particularmente en la etapa de aprendizaje automático que, como su nombre lo indica, es la etapa donde el algoritmo aprende que una muestra con cier- to conjunto de características generalmente está asociado a cierta clase, y en consecuencia debe asignarse a ella. Para realizar tareas de clasificación de manera automática, existen diferentes clasificadores que utilizan las características extraídas, mediante el procesamiento de la imagen, por ejemplo, en tareas de clasificación/determinación del estado de maduración en frutos.

Los clasificadores bayesianos son clasificadores populares y muy simples utilizados en diferentes ámbitos de aplicación. Muy a menudo se aplican como modelo de referencia para otros clasificadores. Una de las principales hipótesis que constituyen el enfoque bayesiano para la clasificación es la independencia de los atributos. Sin embargo, aunque en problemas reales esto no puede ser siempre verdad, el rendimiento del clasificador bayesiano es bueno.

El clasificador bayesiano se basa en la regla de la probabilidad condicional de Bayes (1) (Barón, 2014). Donde $p\left(c_{j} \mid d\right)$ es la probabilidad de la instancia $d$ de pertenecer a la clase $c_{j^{\prime}}$ es $p\left(d \mid c_{j}\right)$ la probabilidad de obtener $d$ en la clase $c_{j}, p\left(c_{j}\right)$ es la probabilidad de ocurrencia de la clase $c_{j}$, y $p(d)$ es la probabilidad de ocurrencia de la instancia $d$.

$$
p\left(c_{j} \mid d\right)=\frac{p\left(d \mid c_{j}\right) p\left(c_{j}\right)}{p(d)}
$$

El método de los k-vecinos más cercanos, K-NN, es un algoritmo de clasificación muy conocido y utilizado generalmente en el reconocimiento de patrones. En el algoritmo original de K-NN, el objeto se asigna a la clase que presente una mayor correspondencia de acuerdo con sus $k$ vecinos más cercanos, KNNs. Esta correspondencia es definida en función de las distancias entre la instancia y sus $k$ vecinos (Liu, Pan, \& Dezert, 2013). Una red neuronal del tipo perceptrón multicapa, MLP, de alimentación hacia adelante con una sola capa oculta se compone de una capa o conjunto de entradas, una sola capa de unidades o neuronas ocultas y una capa de salidas. Los pesos de entra- 
da conectan con cada una de las neuronas de la capa oculta y los pesos de salida conectan con la activación de cada una de las salidas. Los datos de entrenamiento son descritos por el conjunto de elementos en la capa de entrada lo cual conforma un vector de entrada $\mathrm{N}$-dimensional, y los vectores de salida M-dimensionales en función de las clases o conjuntos deseados (Malaur, Manry, \& Jesudhas, 2015). Ellas se han utilizado ampliamente en aplicaciones de regresión y clasificación en áreas como: estimación de parámetros, análisis de documentos y reconocimiento, finanzas, fabricación y minería de datos.

\subsection{Análisis de colorimetría}

El color es un atributo de las plantas y sus frutos $y$, en los procesos de identificación, puede también ayudar a diagnosticar el estado de desarrollo (Simpson, 2011). Esta característica es representada cuantitativamente mediante el índice de color, el cual es un número adimensional que relaciona los distintos valores de las componentes de color (Ej.: RGB - Componente R, G y B; independientemente).

El análisis de colorimetría clásico se realiza bajo condiciones ambientales controladas, generalmente dentro de un recinto cerrado, con temperatura estable y luminosidad solar limitadas. El desarrollo de la prueba consiste en la obtención de valores de la epidermis, por medio de un colorímetro, que arroja datos en el espacio de color CIEL* $a^{*} b^{*}$ (en coordenadas $L^{*}, a^{*}$ y b*). El método necesita una ligera penetración al fruto para obtener los valores de color sobre la epidermis.

Los parámetros $I^{*}, a^{*}$ y $b^{*}$ describen el estado de coloración del fruto en puntos específicos de la epidermis. Estos valores definen luminosidad, pureza y tono de color respectivamente, de verde a rojo (Eje $a^{*}$ ) y de azul a amarillo (Eje $b^{*}$ ). El índice de color es entonces una relación entre los valores obtenidos para cada fruto, al promediar los datos de 3 o más puntos sobre la superficie del fruto. Existen varias relaciones matemáticas que permi- ten obtener el índice de color, el cual puede ser calculado como se muestra en la ecuación (2).

$$
I C=\frac{a * 1000}{l * b} .
$$

Por otro lado, el índice de madurez es definido para cada fruto por medio de la relación entre la dulzura y la acidez. Al relacionarlo con el índice de color, este puede ser obtenido mediante un ajuste matemático distinto para cada fruto. La ecuación que representa el índice de color, busca entonces la variación más relevante entre los diferentes valores $\left(I^{*}, a^{*}, b^{*}\right)$, con el objetivo de encontrar una óptima representación del color en el fruto.

A partir de allí es posible desarrollar un método computacional que determine el estado de maduración de un fruto de feijoa por un método no destructivo, utilizando técnicas de visión por computador. La posibilidad de obtener automáticamente los valores en el espacio de color CIELab para una cantidad mayor de puntos sobre el fruto, puede llegar a representar de mejor forma la relación color-madurez. Las técnicas de visión por computador contribuyen entonces, a la obtención de las características de un objeto, expresadas cuantitativamente, en este caso las coordenadas de distintos espacios de color, sobre frutos de feijoa.

\subsection{Metodología}

Frutos de feijoa provenientes del departamento de Cundinamarca, Colombia, fueron utilizados para el estudio. Se determinaron las características fisicoquímicas de la feijoa que inciden en la clasificación, según estados de madurez. Ingenieros agrícolas y personal experto clasificaron los frutos realizando una serie de pruebas de laboratorio: sólidos solubles totales ('brix), firmeza de corteza externa del fruto, color, acidez, $\mathrm{pH}$, índice de madurez, respiración, peso, volumen, diámetro menor, diámetro mayor, forma, tamaño. Las pruebas fueron realizadas sobre un conjunto de 156 feijoas en los laboratorios de la Universidad Nacional de Colombia, las cuales fueron fotografiadas en un re- 
cinto cerrado con un sistema de iluminación superior atenuado utilizando papel mantequilla, justo antes de realizar el conjunto de pruebas descrito. Esta caracterización fisicoquímica fue fundamental con el fin de combinar diferentes criterios subjetivos con criterios objetivos y clasificar adecuadamente cada uno de los frutos.

El sistema de visión por computador desarrollado, se basó en tres etapas fundamentales, necesarias para la segmentación y clasificación, por color, de los 156 frutos de feijoa. Inicialmente, las imágenes se adecuaron, realizando un procesamiento de imagen mediante un algoritmo computacio- nal que permitiera la eliminación de las sombras y la identificación del fruto dentro de la escena. Posteriormente, cada imagen fue transformada a 4 espacios de color (RGB, ClELab, YCbCr y HSV), y subdividida en cada uno de sus planos. En total se obtuvieron 12 características al realizar el procedimiento anterior. La metodología puede ser observada en la figura 1. Las imágenes de los frutos fueron adquiridas por una cámara Canon Powershot A590 IS en la longitud de onda del espectro visible, en color RGB y una resolución de 8 Mega-píxeles. Los algoritmos fueron desarrollados en Linux Ubuntu 14.04, utilizando las librerías de software libre OpenCV 2.4 en el entorno Qt creator 4.8.

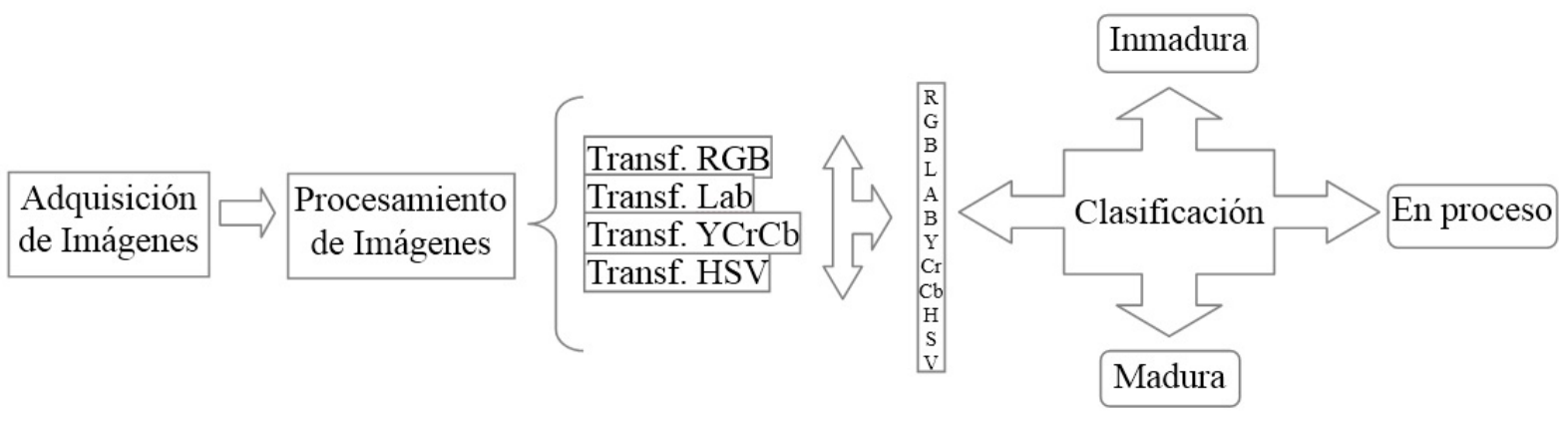

Figura 1. Metodología utilizada para clasificar feijoa a partir de diferentes espacios de color.

El sistema de visión por computador clasifica en las tres fases del proceso de madurez: inmaduro, en proceso de maduración y maduro. Se incluyeron los valores promedio de 4 espacios de color: RGB, CIELab, YCbCr y HSV, en cada uno de sus 3 planos de manera independiente, con la intención de dar mayores herramientas al sistema para la clasificación. Mediante el análisis de componentes principales, fue posible reducir características en el sistema.

La figura 2 ejemplifica las imágenes utilizadas para el proceso de visión por computador. En estas se aprecia que el color verde (negro en escala de grises) va disminuyendo su intensidad, y los colores amarillo y blanco (blanco en la escala de grises) aparecen sobre el fruto, en el momento de la maduración. Los problemas de luminosidad y sombras, afectan la segmentación de puntos del fruto, con color uniforme. Como solución se implementó un algoritmo de eliminación de sombras, el cual se basa en un sesgo del color rojo para la imagen HSV de la escena donde se encuentra el fruto. De igual manera, se utilizó la técnica de filtro morfológico, con el objetivo de diferenciar y obtener el fruto de manera independiente. 

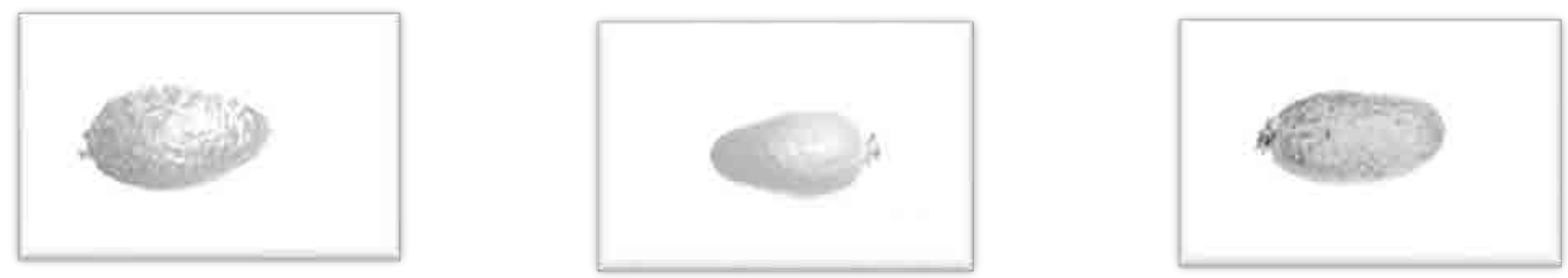

Figura 2. Muestras del banco de imágenes utilizadas para desarrollar el sistema de visión por computador, para cada una de las clases propuestas. De izquierda a derecha: feijoa inmadura, feijoa en proceso de maduración y feijoa madura.

Extraídas las características de color sobre la imagen de la feijoa segmentada, se evaluaron tres clasificadores, con el objetivo de obtener una respuesta del estado de maduración del fruto, en función de la imagen. El que mejor desempeño presentó fue la red neuronal artificial. Adicionalmente, se realizó un análisis por componentes principales (PCA), con el objetivo de eliminar datos redundantes, que estuvieran arrojando información no útil al sistema de clasificación. Para el proceso de clasificación fueron utilizados 3 clasificadores: bayesiano, k-vecinos más cercanos y redes neuronales artificiales.

Luego de la subdivisión por planos, se extrajeron las características de forma independiente $(R, G$, $B, Y, C b, C r, L, a, b, H, S, V)$. Se procedió al entrenamiento, prueba y evaluación de los 3 clasifica- dores. Se utilizaron 111 imágenes de frutos de feijoa, para entrenar cada uno de los clasificadores, 37 por cada una de las clases. Como validación se utilizaron 15 imágenes por clase (45 imágenes). En total se utilizaron 156 imágenes, sumando los datos de entrenamiento y prueba.

\section{Resultados y discusión}

Al finalizar el procesamiento de las imágenes, se obtiene una imagen que contiene solo puntos de color, sin influencia de luminosidad, sombras u otros elementos de la escena. La figura 3, muestra los tres pasos fundamentales realizados. La imagen original capturada en la escena, la aplicación del filtro morfológico, la identificación de la máscara que se aplicará a la imagen adquirida, y finalmente la segmentación del fruto.

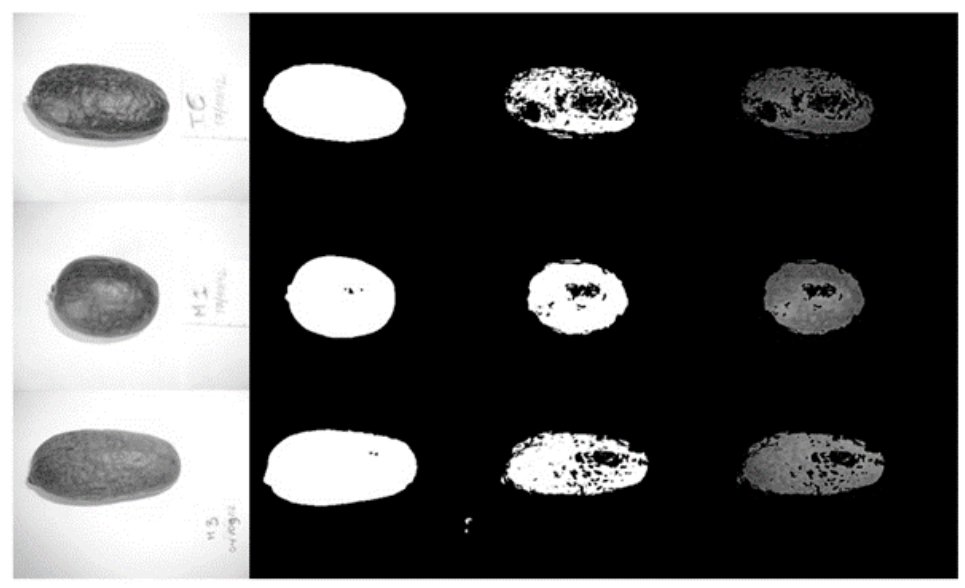

Figura 3. Resultado del procesamiento de imagen y segmentación del fruto para cada una de las clases propuestas. 
Las zonas descartadas en la imagen, que se encuentran dentro de la región del fruto de feijoa, en general, poseen mucho brillo y no aportan en la determinación del estado de desarrollo. Lo anterior debido a los efectos de iluminación provocados por el dispositivo de adquisición de las imágenes, que afectó el color del fruto en algunos puntos específicos. Posteriormente, según la metodología planteada en la figura 1, se obtuvieron las transformaciones de imagen segmentada, en los espacios de color: RGB, YCbCr, Lab y HSV. Las imágenes de cada espacio de color, se subdividieron en cada plano.

El primer clasificador evaluado fue el sistema bayesiano, utilizando tanto las 12 características de cada uno de los espacios de color, como de las 3 características arrojadas por PCA. En las tablas 1 y 2 se puede observar la comparación entre los dos sistemas bayesianos en términos estadísticos. También se presentan los datos obtenidos del análisis estadístico del funcionamiento del clasificador.

Los criterios utilizados, se resumen en: Error Medio (EM), Precisión, Sensitividad, Especificidad, Proporción de falsa aceptación y Exactitud, donde este último determina la calidad del clasificador analizado. El sistema bayesiano utilizando las 3 características principales arrojadas por PCA presenta una mejora del $4,43 \%$ pasando de $86,67 \%$ a $91,11 \%$ en la exactitud de la clase Madura, respecto al sistema que utiliza las 12 características.

\begin{tabular}{|c|c|c|c|c|}
\hline \multicolumn{5}{|c|}{ Matriz de confusión y análisis de error } \\
\hline & Inmadura & & & Madura \\
\hline Inmadura & $40 \%$ & & & $20 \%$ \\
\hline En maduración & $0 \%$ & & & $20 \%$ \\
\hline \multirow[t]{2}{*}{ Madura } & $0 \%$ & & & $100 \%$ \\
\hline & VA & VR & FA & FR \\
\hline Inmadura & $13 \%$ & $67 \%$ & $0 \%$ & $20 \%$ \\
\hline En maduración & $27 \%$ & $53 \%$ & $13 \%$ & $7 \%$ \\
\hline Madura & $33 \%$ & $53 \%$ & $13 \%$ & $0 \%$ \\
\hline
\end{tabular}

Tabla 1. Evaluación del proceso de clasificación, mediante un sistema Bayesiano, usando 12 características extraídas de los 4 espacios de color.

\begin{tabular}{|c|c|c|c|c|}
\hline \multicolumn{5}{|c|}{ Matriz de confusión y análisis de error } \\
\hline & Inmadura & & & Madura \\
\hline Inmadura & $47 \%$ & & & $20 \%$ \\
\hline En maduración & $7 \%$ & & & $7 \%$ \\
\hline \multirow[t]{2}{*}{ Madura } & $0 \%$ & & & $100 \%$ \\
\hline & VA & VR & FA & $\mathbf{F R}$ \\
\hline Inmadura & $16 \%$ & $64 \%$ & $2 \%$ & $18 \%$ \\
\hline En maduración & $29 \%$ & $56 \%$ & $11 \%$ & $4 \%$ \\
\hline Madura & $33 \%$ & $58 \%$ & $9 \%$ & $0 \%$ \\
\hline
\end{tabular}




\section{Análisis estadístico}

\begin{tabular}{|c|c|c|c|}
\hline & Inmadura & En maduración & Madura \\
\hline Error medio & 0.20 & 0.20 & 0.13 \\
\hline Precisión & 1.00 & 0.67 & 0.71 \\
\hline Sensitividad & 0.40 & 0.80 & 1.00 \\
\hline Especificidad & 1.00 & 0.80 & 0.80 \\
\hline Prop. Fa & 0.00 & 0.20 & 0.20 \\
\hline Exactitud & 80.00 & 80.00 & 86.67 \\
\hline \multicolumn{4}{|c|}{ Análisis estadístico } \\
\hline & Inmadura & En maduración & Madura \\
\hline Error medio & 0.20 & 0.20 & 0.09 \\
\hline Precisión & 0.88 & 0.67 & 0.79 \\
\hline Sensitividad & 0.47 & 0.80 & 1.00 \\
\hline Especificidad & 0.97 & 0.80 & 0.87 \\
\hline Prop. Fa & 0.03 & 0.20 & 0.13 \\
\hline Exactitud & 80.00 & 80.00 & 91.11 \\
\hline
\end{tabular}

Tabla 2. Evaluación del proceso de clasificación, mediante un sistema Bayesiano, usando las 3 características más representativas arrojadas por PCA.

De igual manera, se evaluaron los clasificadores k-vecinos más cercanos utilizando tanto las 12 características de cada uno de los espacios de color, como de las 3 características arrojadas por PCA. En las tablas 3 y 4 se puede observar la comparación entre los dos sistemas k-vecinos más cercanos en términos estadísticos.

\begin{tabular}{cccc}
\hline & \multicolumn{2}{c}{ Matriz de confusión y análisis de error } & Madura \\
\hline Inmadura & En maduración & \\
& $33 \%$ & $27 \%$ & $40 \%$ \\
En maduración & $7 \%$ & $73 \%$ & $20 \%$
\end{tabular}


Madura

Inmadura

En maduración

Madura
$7 \%$

Va

$11 \%$

$24 \%$

$31 \%$
$0 \%$

$\mathbf{V r}$

$62 \%$

$58 \%$

$47 \%$
$93 \%$

Fa Fr

$4 \%$

$22 \%$

$9 \%$

$9 \%$

$20 \%$

\section{Análisis estadístico}

Inmadura En maduración Madura

$\begin{array}{cccc}\text { Error medio } & 0.27 & 0.20 & 0.22 \\ \text { Precisión } & 0.71 & 0.67 & 0.61 \\ \text { Sensitividad } & 0.33 & 0.80 & 0.93 \\ \text { Especificidad } & 0.93 & 0.80 & 0.70 \\ \text { Prop. Fa } & 0.06 & 0.20 & 0.30 \\ \text { Exactitud } & \mathbf{7 3 . 3 3} & \mathbf{8 0 . 0 0} & \mathbf{7 7 . 7 8}\end{array}$

Tabla 3. Evaluación del proceso de clasificación, mediante un sistema k-vecinos más cercanos, usando 12 características extraídas de los 4 espacios de color.

\begin{tabular}{|c|c|c|c|c|}
\hline \multicolumn{5}{|c|}{ Matriz de confusión y análisis de error } \\
\hline & Inmadura & & & Madura \\
\hline Inmadura & $47 \%$ & & & $27 \%$ \\
\hline En maduración & $0 \%$ & & & $7 \%$ \\
\hline \multirow[t]{2}{*}{ Madura } & $7 \%$ & & & $93 \%$ \\
\hline & Va & $\mathbf{V r}$ & $\mathbf{F a}$ & $\mathbf{F r}$ \\
\hline Inmadura & $16 \%$ & $64 \%$ & $2 \%$ & $18 \%$ \\
\hline En maduración & $31 \%$ & $58 \%$ & $9 \%$ & $2 \%$ \\
\hline Madura & $31 \%$ & $56 \%$ & $11 \%$ & $2 \%$ \\
\hline
\end{tabular}

\section{Análisis estadístico}


Inmadura

0.20

0.88

0.47

0.97

0.03
En maduración

Madura

Error medio

Precisión

Especificidad

Prop. Fa
0.13

0.74

0.93

0.83

0.16

80.00

\section{Exactitud}

80.00

\subsection{7}

Tabla 4. Evaluación del proceso de clasificación, mediante un sistema k-vecinos más cercanos, usando las 3 características más representativas arrojadas por el análisis por PCA.

El sistema k-vecinos más cercanos utilizando las 3 características principales arrojadas por PCA presenta una mejora superior al $7 \%$ en la exactitud de las clases inmadura y madura, respecto al sistema que utiliza las 12 características; pasando del $73 \%$ y $78 \%$ al $80 \%$ y $86 \%$, respectivamente. La clase en proceso de maduración posee una exactitud del $80 \%$ para los dos casos.
En el caso de la red neuronal artificial, el número de neuronas de la capa oculta fue evaluado con el fin de evidenciar la precisión del sistema. Las figuras 4 y 5 muestran las diferencias entre los resultados obtenidos al entrenar la red neuronal, con las 12 características extraídas, y el posterior análisis con PCA donde se redujo el número de características a tres. Estas pruebas, permiten identificar la estructura de la red neuronal artificial que produce el mejor resultado de clasificación.

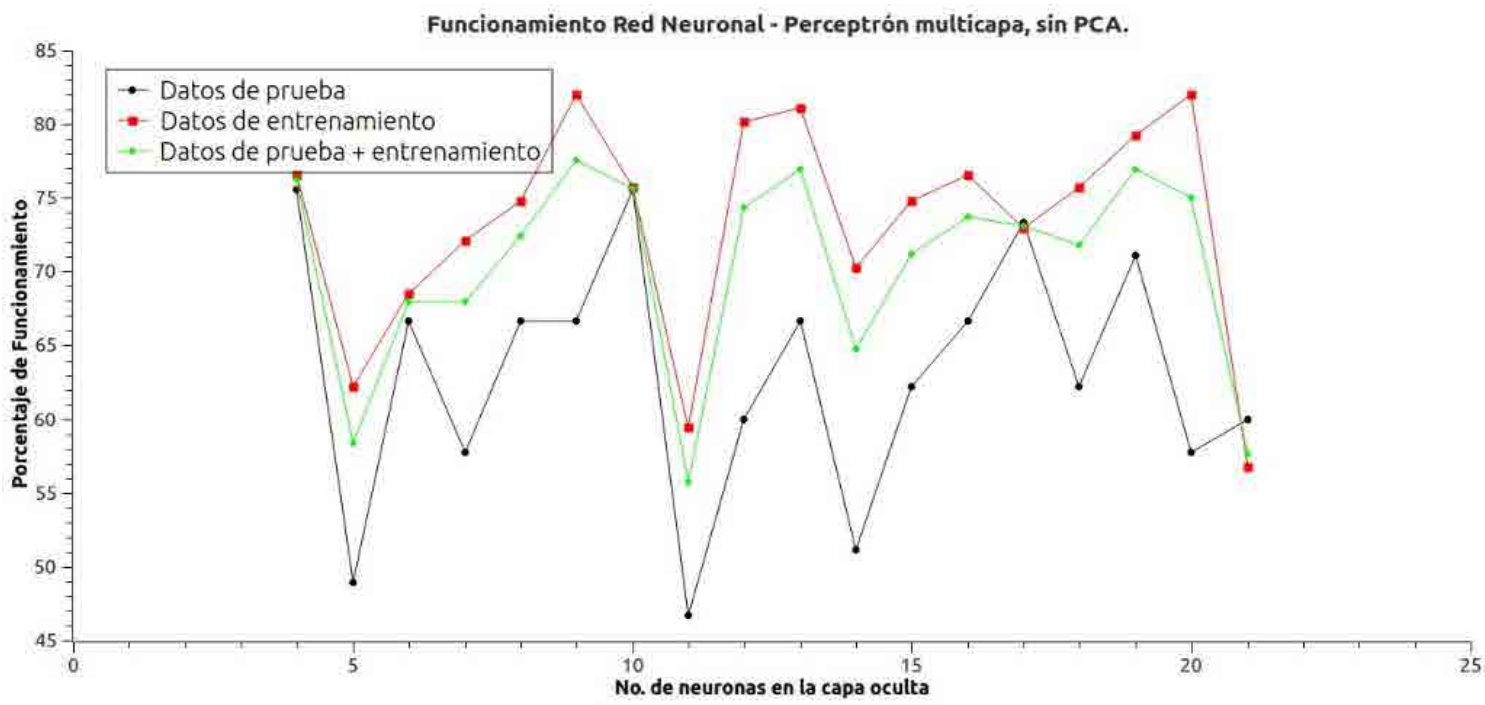

Figura 4. Funcionamiento de la red neuronal - perceptrón multicapa sin aplicar PCA, en función del número de neuronas de la capa oculta. Finalización por criterio de 100 iteraciones o epsilon 0.001. 


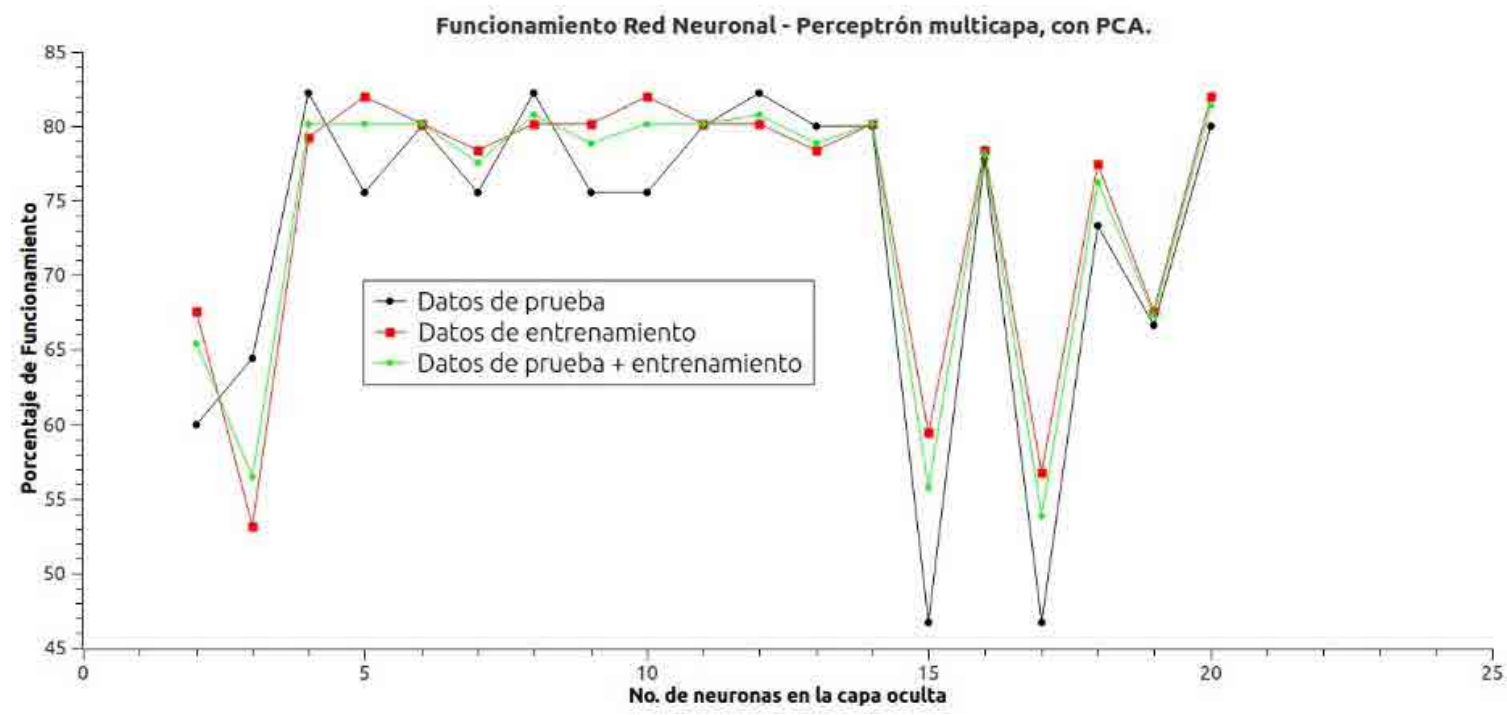

Figura 5. Funcionamiento de la red neuronal - perceptrón multicapa después de aplicar PCA, en función del número de neuronas de la capa oculta. Finalización por criterio de 100 iteraciones o epsilon 0.001.

Al comparar el porcentaje de acierto al clasificar los frutos de feijoa, se observa la variación al utilizar las 12 características o solo tres, obtenidas por PCA; la respuesta del sistema arroja un $85 \%$ aproximado de acierto en la red neuronal con las tres características, mientras que usando las 12 características extraídas el porcentaje es del 65\%. Comparando porcentualmente, la exactitud por clase, se obtiene la mejor respuesta respecto al número de neuronas en la capa oculta, para los sistemas descritos en las figuras 4 y 5 . Es decir, 4 neuronas en la capa oculta para el sistema que utiliza las 12 características, y 12 neuronas en la capa oculta para el sistema que usa solo las 3 características relevantes.
En las tablas 5 y 6 se observan las comparaciones realizadas entre los clasificadores de la red neuronal artificial, antes y después de aplicar PCA, donde se puede evidenciar que la respuesta del sistema, al eliminar la información redundante, es bastante mejor, que en el sistema entrenado con la totalidad de las características. En general, la proporción de falsa aceptación para el clasificador, es baja, en la clase inmadura. Sin embargo, esta clase presenta los menores valores de respuesta en exactitud. En el caso de la red neuronal utilizando PCA, la proporción de falsa aceptación es nula pero se aumenta ligeramente la exactitud para la clase inmadura.

\section{Matriz de confusión y análisis de error}

\section{Inmadura}

$53 \%$

$13 \%$
En maduración

$27 \%$
$20 \%$

Madura
En maduración 


\begin{tabular}{|c|c|c|c|c|}
\hline \multirow[t]{2}{*}{ Madura } & $0 \%$ & \multicolumn{2}{|c|}{$0 \%$} & $100 \%$ \\
\hline & Va & $\mathbf{V r}$ & $\mathbf{F a}$ & Fr \\
\hline Inmadura & $18 \%$ & $62 \%$ & $4 \%$ & $16 \%$ \\
\hline maduración & $24 \%$ & $58 \%$ & $9 \%$ & $9 \%$ \\
\hline Madura & $33 \%$ & $56 \%$ & $11 \%$ & $0 \%$ \\
\hline
\end{tabular}

\section{Análisis estadístico}

Inmadura

0.20

0.80

0.53

0.93

0.07

80.00
En maduración

0.18

0.73

0.73

0.87

0.13

82.22
Madura

0.11

0.75

1.00

0.83

0.17

88.89

Tabla 5. Evaluación del proceso de clasificación, utilizando una red neuronal - perceptrón multicapa, usando 12 características extraídas de los 4 espacios de color.

En las clases en proceso de maduración y madura, la exactitud mejora respecto del clasificador utilizado. El valor de exactitud para la clase en proceso de maduración utilizando la red neuronal con PCA, posee un valor aproximado de $89 \%$ y para el caso de la clase madura, de aproximadamente $93 \%$. Finalmente, la respuesta del sistema utilizando análisis por componentes principales, y una red neuronal, otorga una exactitud de $82.22 \%, 88.89 \%$ y $93.33 \%$ para cada una de las clases, inmadura, en proceso de maduración, y madura, respectivamente.

\section{Matriz de confusión y análisis de error}

\section{Inmadura}

$47 \%$
En maduración

$33 \%$
Madura

$20 \%$ 
En maduración

Madura

Inmadura

En maduración

Madura
$0 \%$

$0 \%$

Va

$16 \%$

$33 \%$

$33 \%$
$100 \%$

$0 \%$

$\mathbf{V r}$

$67 \%$

$56 \%$

$60 \%$
$0 \%$

$100 \%$

$\mathrm{Fa}$

$\mathbf{F r}$

$0 \%$

$18 \%$

$11 \% \quad 0 \%$

$7 \% \quad 0 \%$

\section{Análisis estadístico}

Inmadura En maduración Madura

Error medio

Precisión

Sensitividad

Especificidad

Prop. Fa

Exactitud
0.18

1.00

0.47

1.00

0.00

82.22
0.11

0.07

0.83

1.00

0.90

0.10

0.17

93.33

Tabla 6. Evaluación del proceso de clasificación, utilizando una red neuronal - perceptrón multicapa, usando las 3 características más representativas arrojadas por el análisis por PCA.

El sistema implementado por medio de la metodología planteada, bajo los trabajos realizados para otro tipo de frutos y análisis de frutas, responde de manera adecuada para el caso de la feijoa. Cabe resaltar que el escenario utilizado para la feijoa, disminuye los procesos invasivos que se llevan a cabo para obtener un índice de madurez experimental del fruto, junto con la dificultad en la uniformidad del tono verde presente siempre en el fruto, a lo largo de su proceso de maduración.

\section{Conclusiones}

Se presentó un sistema para identificar el estado de madurez a partir del índice de color, para feijoa -Acca sellowiana-, por medio de una imagen. Debido a la constancia en la tonalidad verde de este fruto, es difícil obtener su índice de color a partir de umbrales entre las clases. La metodología implementada y el análisis de colorimetría por visión por computador contribuyen a resolver la 
problemática de caracterización rápida, eficiente y óptima de frutos exóticos, propios de Colombia y países vecinos.

El proceso de madurez de la feijoa se categorizó en tres fases: inmadura, en maduración y madura. La fase en maduración otorga una mayor robustez al sistema y permite obtener una respuesta más próxima al estado real del fruto. El procesamiento de imágenes, en la metodología propuesta, responde de manera exacta. La eliminación de ruidos propios de la luminosidad, realizando procesos de segmentación y separación en planos de color, otorga las características que de manera más representativa dan información sobre el estado de maduración del fruto. Aunque algunos de los espacios de color se encuentran relacionados, el análisis por componentes principales permitió reducir el sistema de 12 a 3 características, aumentando la proporción de exactitud en el sistema. La implementación de una red neuronal, del tipo perceptrón multicapa, como clasificador de frutos de feijoa, permite la clasificación de manera automática, con un porcentaje cercano al $90 \%$.

Como trabajo futuro, se plantea analizar frutos cosechados en una misma instancia de tiempo y realizar el proceso de análisis poscosecha paralelo al sistema de visión por computador, para poder obtener un estado exacto de maduración (en semanas); así como mejorar el proceso de adquisición de imágenes, para disminuir los efectos iluminantes. Aunque las imágenes y sus categorías fueron identificadas por expertos, es necesario llevar este análisis poscosecha para garantizar de manera exhaustiva su validez al correlacionar aspectos físicos y químicos.

\section{Referencias}

Abdullah, M.Z., Mohamad-Saled, J., Fathinul-Syahir, A.S., \& Mohd-Azemi, B.M.N. (2006). Discrimination and classification of fresh-cut starfruits (Averrhoa carambola L.) using automated machine vision system. Journal of Food Engineering, 76 (4), 506-523. doi: 10.1016/j.jfoodeng.2005.05.053
Arivazhagan, S., Newlin, R., Selva, S., \& Ganesan L. (2010). Fruit Recognition using color and texture features. Journal of Emerging Trends in Computing and Information Sciences, 1 (2), 90-94. Recuperado de: http://www.cisjournal.org/archive/vol1 no1/vol1no1_12.pdf

Baron, G. (2014). Influence of data discretization on efficiency of Bayesian classifier for authorship attribution. Procedia Computer Science, 35, 1112-1121. doi: 10.1016/j.procs.2014.08.201

Blasco, J., Aleixos, N., \& Moltó E. (2003). Machine vision system for automatic quality grading of fruit. Biosystems Egineering, 85 (4), 415-423. doi: /10.1016/ S1537-5110(03)00088-6

Bustamante-Zapata, L.F., Porto-Pérez, I.A., \& Hernández-Taboada, F. (2013). Gestión estratégica de las áreas funcionales de la empresa: una perspectiva competitiva internacional. Revista de Investigación, Desarrollo e Innovación, 4 (1), 56-68. doi: $10.19053 / 20278306.2607$

Cárdenas, J. A., \& Prieto-Ortíz, F. A. (2015). Diseño de un algoritmo de corrección automática de posición para el proceso de perforado $\mathrm{PCB}$, empleando técnicas de visión artificial. Revista de Investigación, Desarrollo e Innovación, 5 (2), 107-118. doi: $10.19053 / 20278306.3720$

Cerón-Correa, A., Salazar-Jiménez, A. E., \& Prieto-Ortiz, F. A. (2013). Reconocimiento de rostros y gestos faciales mediante un análisis de relevancia con imágenes 3D. Revista de Investigación, Desarrollo e Innovación, 4 (1), 7-20. doi:10.19053/20278306.2563

Danti, A., \& Suresha. (2012). Segmentation and classification of raw arecanuts based on three sigma control limits. Procedia Technology, 4, 215-219. doi: 10.1016/j.protcy.2012.05.032

Du, C.J., \& Sun D.W. (2006). Learning techniques used in computer vision for food quality evaluation: a review. Journal of Food Engineering, 72 (1), 39-55. doi: 10.1016/j.jfoodeng.2004.11.017 
East, A.R., Trejo-Araya, X.I., Hertog, M.L.A.T.M, Nicholson, S.E., \& Mawson A.J. (2009). The effect of controlled atmospheres on respiration and rate of quality change in 'Unique' feijoa fruit. Postharvest Biology and Technology, 53 (1-2), 66-71. doi: 10.1016/j.postharvbio.2009.02.002

García, Y., García, A., Hernández, A., \& Pérez J. (2011). Estudio de la variación del índice de color durante la conservación de la piña variedad Cayena Lisa a temperatura ambiente. Revista Ciencias Técnicas Agropecuarias, 20 (4), 12-16. Recuperado de: http:// scielo.sld.cu/scielo.php?script=sci_arttext\&pi$\mathrm{d}=$ S2071-00542011000400002

Kondo, N., Ahmad, U., Monta, M., \& Murase, H. (2000). Machine vision based quality evaluation of lyokan orange fruit using neural networks. Computers and electronics in agriculture, 29 (1-2), 135-147. doi: 10.1016/S0168-1699(00)00141-1

León, K., Mery, D., Pedreschi, F., \& León J. (2006). Color measurement in $L^{*} a^{*} b^{*}$ units from RGB digital images. Food Research International, 39 (10), 1084-1091. doi: 10.1016/j.foodres.2006.03.006

Liu, Z., Pan, Q., \& Dezert, J. (2013). A new belief-based K-nearest neighbor classification method. Pattern Recognition, 46 (3), 834-844. doi: 10.1016/j.patcog.2012.10.001

Malaur, S., Manry, M., \& Jesudhas, P. (2015). Multiple optimal learning factors for the multi-layer perceptron. Neurocomputing, 149 (Parte C), 1490-1501. doi: 10.1016/j.neucom.2014.08.043

Manickavasagana, A., Al-Mezeinia, N.K., \& Al-Shekaili, H.N. (2014). RGB color imaging technique for grading of dates. Scientia Horticulturae, 175, 87-94. doi: 10.1016/j.scienta.2014.06.003

Ohali, Y.A. (2011). Computer vision based date fruit grading system: Design and implementation. Journal of King Saud University - Computer and Information Sciences, 23 (1), 29-36. doi: 10.1016/j.jksuci.2010.03.003
Sanabria-Neira, N. C., \& Puentes-Montañez, G. A. (2011). Sistema de gestión de calidad para el agronegocio de la uchuva en el municipio de Ventaquemada. Revista de Investigación, Desarrollo e Innovación, 1 (2), 28-39. Recuperado de: http://revistas.uptc.edu. co/revistas/index.php/investigacion_duitama/article/view/1300

Sandoval, Z. (2005). Caracterización y clasificación de café cereza usando visión artificial (Tesis de Maestría). Universidad Nacional de Colombia - Sede Manizales, Colombia.

Simpson, N. (2011). Color and contemporary digital botanical illustration. Optics \& Laser Technology, 43 (2), 330-336. doi: 10.1016/j.optlastec.2008.12.014

Thompson, K. (1998). Tecnología post-cosecha de frutas y hortalizas [CD-ROM]. Colombia: Distrididactika Ldta.

Torres, R., Montes, E., Pérez, O., \& Andrade, R. (2013). Relación del color y del estado de madurez con las propiedades fisicoquímicas de frutas tropicales. Información Tecnológica, 24 (3), 51-56. doi: 10.4067/ S0718-07642013000300007

Torres-Barahona, E. A., León-Medina, J. X., \& Torres-Díaz, E. (2012). Sistema de posicionamiento aplicado a la técnica de impresión 3D modelado por deposición fundida. Revista de Investigación, DesarroIlo e Innovación, 3 (1), 25-32. Recuperado de http:// revistas.uptc.edu.co/revistas/index.php/investigacion_duitama/article/view/2135/2091

Vignoni, L., Cesari, R., Forte, M., \& Mirabile, M. (2006). Determinación de índice de color en ajo picado. Información Tecnológica, 17 (6), 63-67. doi: 10.4067/ S0718-07642006000600011

Yama K.L., \& Papadakis S.E. (2004). A simple digital imaging method for measuring and analyzing color of food surfaces. Journal of Food Engineering, 61 (1), 137-142. doi: 10.1016/S0260-8774(03)00195-X 